\title{
Linx
}

Revue des linguistes de l'université Paris X Nanterre

$10 \mid 1998$

L'indicible et ses marques dans l'énonciation

\section{Chemins de sens en lecture}

\section{Cristina Casadei Pietraróia}

\section{(2) OpenEdition}

Journals

Édition électronique

URL : http://journals.openedition.org/linx/973

DOI : $10.4000 /$ linx. 973

ISSN : 2118-9692

\section{Éditeur}

Presses universitaires de Paris Nanterre

\section{Édition imprimée}

Date de publication : 1 juillet 1998

Pagination : 87-107

ISSN : 0246-8743

\section{Référence électronique}

Cristina Casadei Pietraróia, «Chemins de sens en lecture », Linx [En ligne], 10 | 1998, mis en ligne le 04 juillet 2012, consulté le 30 avril 2019. URL : http://journals.openedition.org/linx/973 ; DOI : 10.4000/ $\operatorname{lin} x .973$

Ce document a été généré automatiquement le 30 avril 2019.

Département de Sciences du langage, Université Paris Ouest 


\title{
Chemins de sens en lecture
}

\author{
Cristina Casadei Pietraróia
}

\section{Introduction}

1 «Tout le bois l'attendait. Des mésanges, sceptiques ou qui feignaient de l'être, allaient de branche en branche, faussement indifférentes. Il n'existe pas. On laissait dire. Mais ce coucou très vieux disait l'avoir connu toujours. Il viendrait, comme chaque année. Les mésanges riaient. Elles tenaient d'un gros chêne qu'il était bien venu, parfois, mais qu'il avait changé. Etait-ce seulement le même? Les hommes se ressemblent tant. Légendes, disait le chêne, et légendes récentes; il n'y a pas si longtemps, personne n'en parlait. Mais les chênes comptent en siècles. ${ }^{1}$

Dire ce que l'on a pu comprendre de la lecture de cet extrait, début d'une nouvelle, voilà un exercice bien classique d'une classe de langue et qui ne pose pas beaucoup de problèmes. Cependant, répondre à la question "Comment a-t-on compris cet extrait?", nous met déjà dans une autre situation. On peut, bien sûr, indiquer quelques chemins de lecture tels que le repérage du réseau anaphorique, le rapprochement des éléments appartenant aux mêmes champs lexicaux, l'attention aux indices temporels, mais ce sont là des stratégies utilisées pour la construction du sens qui peuvent ne pas être exactement les mêmes chemins de sens parcourus par chaque lecteur. Ceux-ci varient selon les représentations du monde, les connaissances linguistiques, culturelles et encyclopédiques de chacun, selon son projet de lecture, son niveau d'attention, son intérêt pour le texte, etc. Et voilà la difficulté de toute recherche portant sur la lecture : peut-on expliciter ces éléments?

3 La lecture, on le sait, c'est de la communication, de l'interaction, du dialogue, mais entre un texte et son lecteur. Créer un tiers élément dans cette communication n'est pas évident: comment expliciter notre compréhension? Comment la transmettre à quelqu'un qui observe du dehors? Quand on essaie de raconter à quelqu'un comment on a compris un texte, on entre déjà dans un autre stade du discours, on passe du compris au rapporté, qui bénéficie, à son tour, d'une série d'autres éléments peut-être absents lors de la compréhension et de la construction premières du sens. Et l'on inaugure une autre 
situation de communication qui n'est plus celle d'un texte et son lecteur, activité unique, privée, peu partageable et maintes fois incommunicable, voire indicible... Comme l'affirme Alberto Manguel dans son Une histoire de la lecture,

«(...) l'histoire de la lecture c'est l'histoire de chacun de ses lecteurs. ${ }^{2}$ »

\section{Saisir l'indicible}

Dans l'espoir de saisir ce formidable indicible, différents courants de recherche qui se penchent sur la lecture ont élaboré des méthodes et des protocoles divers qui vont de la "simple ${ }^{3}$ observation des comportements du lecteur (ses prises de notes, ses hésitations, ses retours en arrière, etc) jusqu'aux méthodes informatisées, telle qu'ECCLA (Evaluation-diagnostic des Capacités Cognitives du Lecteur Adulte ${ }^{4}$ ), en passant par l'étude des mouvements oculaires, les protocoles de pause, le résumé, les tests clos, les questionnaires à choix multiple, etc. Répondant à des conceptions théoriques spécifiques et à des objectifs différents de recherche ${ }^{5}$, ces processus d'investigation de la lecture - à l'oeuvre à partir des années 60 surtout - nous offrent aujourd'hui un panorama assez complet de la compréhension écrite, surtout en langue maternelle. En ce qui concerne la lecture en langue étrangère, pourtant, la situation est tout autre, au moins dans le domaine du Français Langue Etrangère, comme l'affirment S. Moirand e F. Cicurel (1990)6

5 La difficulté de l'investigation a sûrement une influence sur le manque de recherches. Si la variation des chemins de construction du sens et la difficulté à les expliciter sont déjà importantes dans une situation de lecture en langue maternelle, imaginons ce qui se passe en langue étrangère, surtout quand on a affaire à des lecteurs qui maîtrisent peu ou mal la langue en question? Dans ce domaine, nous avons généralement affaire à des sujets qui ont déjà une compétence de compréhension écrite assez bien développée et une expérience langagière construite dans des situations qui nous échappent. En outre, l'apprentissage du nouvel idiome se produit dans des contextes et des situations spécifiques qui produisent des effets peu négligeables pour la lecture (le contexte scolaire, la motivation à l'apprentissage, l'objectif de la lecture, le type de texte présenté à l'étudiant, etc). Comme nous pouvons le constater, les éléments pris en compte lors de la compréhension en lecture (le rôle des représentations mentales, le lexique, le contexte, les hypothèses du lecteur, ses attentes) doublent ainsi d'épaisseur, ce qui ne va pas sans poser de problèmes aux recherches.

On sait aujourd'hui que dans l'activité de compréhension écrite interviennent deux grands types de stratégies, autrefois opposées, mais qui sont extrêmement dépendantes et intégrées : les stratégies ascendantes, ou botton-up et les stratégies descendantes, ou top-down. Les ascendantes sont celles qui partent du texte pour arriver au sens : le lecteur assemble d'abord les syllabes, puis les mots et finalement les phrases et les paragraphes pour arriver au sens global du texte. C'est une lecture par synthèse. Les stratégies descendantes partent, au contraire, du sens général qu'on peut saisir d'un texte, pour arriver à sa structure, ses mots, ses éléments; on part d'une ou de plusieurs hypothèses sur le texte pour arriver aux détails. Ce sont des stratégies extrêment importantes et qui assurent, en outre, l'intégration entre les connaissances personnelles du lecteur et celles apportées par le texte.

7 La didactique des langues - maternelles ou étrangères - a privilégié à des moments différents l'une ou l'autre de ces stratégies. Les méthodes traditionnelles, par exemple, étaient construites à partir des stratégies ascendantes. En revanche, les méthodes basées 
sur l'approche globale (en ce qui concerne les langues étrangères) et les méthodes idéovisuelles (pour la lecture en langue maternelle) ont comme point de départ les stratégies descendantes. Aujourd'hui nous savons que le bon lecteur maîtrise et fait inter-agir les deux types de stratégies en même temps, surtout parce que leur absence (ou leur excès) peut court-circuiter la compréhension. Les bons lecteurs sont ceux qui arrivent à créer et à maintenir sous contrôle ce processus de va-et-vient entre les éléments du texte et les informations que lui, lecteur, possède déjà. Les mauvais lecteurs sont, au contraire, ceux qui ne s'appuient que sur l'un ou l'autre type d'élément ou de stratégie.

Mais le besoin de nouvelles recherches se fait encore sentir, car il est difficile de préciser comment cette interaction a vraiment lieu. L'article qui se présente ici porte justement sur une recherche menée auprès des étudiants universitaires de $\mathrm{FLE}^{7}$ dont l'objectif était de connaître un peu mieux les mécanismes de construction du sens dans une situation de lecture gênée par la faible maîtrise de la langue étrangère. Ses résultats nous ont surpris, car nous avons pu «guetter» l'indicible et formuler des hypothèses pour la compréhension écrite en langue étrangère.

\section{Un rapport annonce...}

9 La recherche en question a été menée auprès de quelques universitaires brésiliens apprenant le français. Neuf étudiants de la première année de français de l'Université de São Paulo ont reçu un article du journal Libération du 09 novembre 1994 concernant les fausses offres d'emploi $^{8}$; comme consigne ils ont eu la tâche de traduire ce texte oralement, en s'arrêtant à chaque problème paru et en essayant d'expliquer au maximum leurs processus d'élucidation/construction du sens. Leur lecture/traduction a été enregistrée sur le moment et n'a pas été précédée d'une lecture silencieuse ou d'un autre travail préalable quelconque. Les lecteurs ne connaissaient pas l'objectif de la recherche, ni le destin de leurs productions. Celles-ci étaient considérées comme un exercice de compréhension en lecture. Ce protocole a été caractérisé, ainsi, par une approche introspective (verbalisation de la pensée au moment de la lecture) et la recherche a été fondamentalement descriptive, sans manipulation ou restructuration de la situation d'enseignement.

10 Ayant comme base théorique les études d'orientation cognitive sur la lecture et les études énonciatives du langage, cette recherche considère la lecture comme une activité de communication, construction et représentation du sens. Les études portant sur l'acquisition lexicale, aussi bien en langue maternelle qu'en langue étrangère, et les travaux de Vygotsky sur l'acquisition du langage ont aidé, à leur tour, dans l'analyse des données qui a consisté, fondamentalement, dans l'analyse du traitement lexical fait par les lecteurs. Une interaction entre les stratégies ascendantes et descendantes a pu être attestée, mais c'est le travail fait par les lecteurs sur le lexique qui a surtout attiré notre attention comme étant l'aspect le plus productif de leur lecture - et, bizarrement, un des aspects les plus négligés de la lecture en langue étrangère ces derniers temps.

11 Pour une bonne compréhension de l'analyse, nous présentons, brièvement, quelques-uns des principaux fondements théoriques qui ont conduit notre recherche.

12 Les études d'orientation cognitive considèrent l'individu humain comme un système de transformation des informations provenant du monde et de la mémoire dans des 
représentations mentales et/ou des actions, et la science cognitive cherche à le décrire selon ses structures et les opérations cognitives employées.

Deux types de structures cognitives sont en générale prises en considération: les représentations-types et les représentations-occurrentes. Les premières décrivent l'état initial de la mémoire, c'est-à-dire, l'état de l'information mémorielle antérieur à son activation pendant le traitement du discours. Comprenant les connaissances linguistiques et les connaissances du monde (des notions générales, des croyances, etc) de l'individu, ce type de représentation est généralement décrit en termes de schémas mentaux. Étudiés selon des perspectives et des dénominations différentes par divers auteurs - scripts (Shank et Abelson), frames (Minsky), scénarios (Sanford et Garrod) - ils peuvent être vus comme « des réseaux sémantiques dont les éléments entretiennent des relations privilégiées du fait de la fréquence et, peut-être, de la nature des co-occurrences» (Fayol, 1992 :74). Plus ou moins abstraits, les schémas mentaux sont organisés de façon hiérarchisée ou non et quelques-uns de leurs éléments sont déterminés par des inférences (des informations absentes du schéma) en fonction de ce qui, en général, arrive dans le contexte socioculturel des individus.

Une fois activées, les structures schématiques vont guider - de manière même contraignante - la construction de la représentation à tous les niveaux, et cela de trois manières : comme base de données dans la compréhension, en tant que filtre dans la sélection des informations qui pourraient venir remplir les lacunes, et en tant que "générateur d'attentes". Les exemples les plus cités, tels que les schémas mentaux sur Noël ou les anniversaires, montrent l'hétérogénéité de ce processus constructif qui ne se vérifie pas seulement entre des groupes sociaux différents, mais d'individu à individu, étant donné que, pour être des représentations, les structures schématiques mentales sont directement liées à l'expérience et à l'histoire culturelle de chaque personne, vécues parfois de la même façon, mais mises en mémoire et récupérées de façon différente.

En ce qui concerne les représentations-occurrentes, elles peuvent être considérées comme des événements mémoriels de durée limitée qui peuvent être intégrés aux représentations-types en fonction de l'importance (en général subjective et inconsciente) attribuée par l'individu. En lecture, par exemple, elles correspondent aux résultats provisoires du traitement textuel qui sont contrôlés et intégrés aux représentations-type.

Les opérations cognitives, de leur côté, définissent les processus de changement des états de l'information qui ont lieu entre la présentation du discours et la construction de la représentation-occurrente. Dans le cas de la lecture/compréhension des textes, on travaille sur les opérations d'identification des unités de surface, la construction en temps réel de la signification locale et globale du texte, la mémorisation, le stockage et le maintien en mémoire de l'information - caractérisés par des modifications passives ou semi-passives (suppressions, substitutions, interférences) - et, finalement, le rappel de l'information sémantique et la production d'un nouveau texte lors de la restitution ou de la formulation des réponses.

17 La compréhension d'un texte est considérée, ainsi, comme un processus extrêmement actif où sont en jeu aussi bien les éléments textuels que les connaissances du lecteur (linguistiques, encyclopédiques, discursives, etc). Elle ne peut, en aucun cas, être associée à un traitement linéaire et additif d'une séquence d'énoncés.

Une conception de cette sorte rejette les modèles essentiellement ascendants ou descendants de lecture et en impose d'autres moins linéaires/hiérarchisés et plus 
flexibles, tels que les modèles connexionnistes (Kintsh, 1988) ou d'activation-diffusion (Le $\mathrm{Ny}, 1989$ ). Pour ceux-ci, toutes les structures et les étapes de la compréhension textuelle se trouvent interconnectées et en constant mouvement d'activation, diffusion, inhibition et intégration, accentuant des éléments déjà existants, ayant recours à d'autres moins disponibles, revenant sur des choix pris :

«(...) tout se modifie sans doute simultanément et, peut-être, en interaction : les signifiants disponibles (lexique...); les signifiés (base de connaissances); les procédures ; les capacités de contrôle, etc. $\rrbracket^{9}$

19 Ces nouveaux modèles, qui attribuent une grande importance aux stages intermédiaires de la transformation de l'information et qui rejettent la linéarisation du processus de traitement du langage, considèrent tous les éléments du stimulus à la fois. Ils ont été le résultat - et ont donné comme résultat - des recherches de plus en plus ponctuelles sur les spécificités de la lecture, depuis les mécanismes d'identification d'un mot jusqu'à la compréhension globale d'un texte, en passant par les mouvements oculaires (les fixations), la vitesse de lecture, le rôle du contexte dans la compréhension écrite et les procédures d'activation du lexique mental. Ce dernier point nous intéresse de façon particulière, car il est directement lié au traitement lexical et il convient de s'y arrêter un peu.

Le lexique mental est une sorte de dictionnaire mental qui comporte tous les mots connus par l'individu, auxquels sont associés des informations orthographiques, phonologiques, syntaxiques et sémantiques. Reconnaître un mot c'est chercher une entrée dans ce dictionnaire et, une fois trouvé, on sait comment le prononcer, à quelle classe lexicale il appartient et ce qu'il signifie. D'après les études faites, un individu adulte aurait un lexique mental d'environ 50.000 mots; ainsi, lors de la lecture d'un de ces mots, il activerait, d'abord, dans son lexique mental, un ensemble de candidats correspondants au stimulus visuel (phase d'identification ou d'accès lexical) et, ensuite, lors du choix d'un de ces candidats, il réaliserait une phase de reconnaissance proprement dite (attribution du sens). Le processus global, connu comme reconnaissance lexicale, présente les « caractéristique suivantes » (Segui, 1992):

- une extrême rapidité et efficacité (un lecteur habile est capable de traiter 5 mots par seconde en moyenne, taux qui peut atteindre un maximum de 15 mots si le lecteur a recours à des procédures techniques) ;

- il s'agit d'un processus automatique et, ce qui est le plus important, irrépressible, c'est que, quand un lecteur voit une séquence de lettres qui constituent un mot, cette séquence sera traitée en tant que mot, en tant qu'unité linguistique avec une forme et un sens ;

- le lecteur n'est conscient que du résultat du traitement et non de son développement actif, caractéristique qui complique énormément la recherche, d'autant que :

1. la présentation d'un mot-stimulus active non seulement sa propre représentation lexicale, mais encore celle d'un ensemble de mots correspondant à leurs voisins orthographiques (Segui, 1992). Ainsi, le mot FOIN a comme voisins COIN, SOIN, LOIN, FOIE. Si l'un de ces mots voisins a une fréquence d'emploi plus grande que le mot-cible, il y aura une augmentation du temps de lecture de ce dernier, puisque le terme de plus grande fréquence sera plus rapidement accessible au système de réponse ;

2. l'identification d'un mot produit aussi une activation involontaire, rapide et faible de ses voisins sémantiques (Pynthe, 1983, Alegria, 1991 entre autres). Si l'un de ces voisins est activé dans les limites du temps nécessaire $(50 \mathrm{~ms})$ on aura une réponse d'identification plus rapide que dans une situation neutre. Ainsi, la reconnaissance du 
mot «hôpital » sera plus immédiate si elle vient après le mot «infirmière » qu'après «musée », par exemple. Il est important de souligner que ce type d'activation a un caractère immédiat, éphémère, involontaire et non sélectif, c'est-à-dire que l'activation s'étend sur tous les voisins sémantiques du mot qui doit être reconnu et ce processus ne comporte aucune charge cognitive ${ }^{10}$.

En résumé, on peut dire que la lecture d'un mot active de façon automatique tous les sens qui lui sont associés. Ensuite, et de façon extrêmement rapide, si le contexte et les indices morpho-syntaxiques le permettent, un sens unique, celui qui s'accorde avec l'ensemble des éléments, est sélectionné. Il est intéressant de noter que les sujets experts maintiennent différentes interprétations d'un item ambigu, alors que les sujets plus faibles semblent procéder à un choix beaucoup plus tôt, faisant ainsi des fautes de compréhension qui les obligent à plusieurs retours en arrière pendant la lecture. Pynte (1983 : 98, à la suite de Carr, 1981) affirme que :

(...) les bons lecteurs sont capables d'envisager simultanément un grand nombre d'interprétations concurrentes tandis que les mauvais lecteurs utiliseraient le contexte pour émettre des hypothèses plus focalisées.

Finalement, les recherches cognitives mettent l'accent sur les stratégies métacognitives employées surtout par les lecteurs experts. Ces stratégies visent la gestion et le contrôle de la situation de lecture et elles sont présentes à tous les niveaux : lexical, syntaxique, sémantique, grammatical, discursif. Ce sont elles qui, par exemple, contrôlent les différents processus de reconnaissance lexicale cités ci-dessus, aidant le lecteur à guider sa compréhension et à finaliser la construction d'une représentation occurrente du texte lu.

\section{...une augmentation des pistes pour la lecture en langue étrangère}

23 Le travail avec des étudiants universitaires apprenant le français nous a effectivement montré l'hétérogénéité et la variété des stratégies et des mécanismes employés pendant la lecture d'un texte. Comme nous l'avons déjà indiqué, le texte utilisé dans notre recherche était un article extrait du journal Libération sur les fausses offres d'emploi. Ce texte avait été choisi pour son niveau de langue et le thème abordé, qui présentait des similitudes avec la situation économique brésilienne, mais, en même temps, faisait référence à des éléments culturels nouveaux qui pouvaient égarer le lecteur inattentif.

etudiants de première année ont enregistré une lecture faite en portugais de ce texte et les item lexicaux qui leur ont posé le plus de problèmes ont été regroupés et analysés. En voici les principaux : emploi / rapport / fausses / offres / chômage / bidon / chômeurs / plaintes / dressés / bilan / escroquerie / verser / sur le sérieux des offres / systèmes de chaînes d'argent / acquitté / réseau / prospecter / des marchands de listes / embaucher / offres d'emploi Minitel / serveurs / fichiers / repiquer.

Il est important d'observer que les item soulignés sont pour la plupart peu transparents par rapport au portugais et plusieurs d'entre eux font référence à des éléments de la culture française inconnus des étudiants brésiliens. La lecture qui en a été faite a pourtant montré une grande variété de traitements et a imposé, tout de suite, la notion de construction : à la recherche de confirmations pour leurs hypothèses de travail et ayant comme objectif de fixer de nouvelles formes et de nouveaux signifiés, les lecteurs ont 
utilisé des mécanismes de traitement lexical assez complexes qui ont démontré leur effort dans la construction d'un sens considéré comme pertinent et valable.

Ainsi, par exemple, plusieurs termes de la langue étrangère ont été traités à partir de leur similitude avec des termes de la langue maternelle, de la langue étrangère elle-même ou d'une autre langue disponible pour le lecteur (anglais, par exemple). Ce mécanisme est assez connu dans la littérature portant sur le traitement lexical en langue étrangère, mais ce qui nous a surpris c'est que les analogies faites, y compris celles erronées, n'étaient que le début d'un processus qui, en général, aboutissait à la construction d'un sens convenable pour le terme analysé. Les exemples le montrent bien :

- rapport a été lu par un étudiant comme recherche (" pesquisa»), puis comme " relatório »; sur ce même terme, un étudiant a fait le chemin suivant : il a d'abord lu le terme rapport comme rapport sexuel (acception qu'il connaissait), ensuite comme cas («casos»), ensuite comme liste (« lista », « listagem ») d'où il a pris le terme « reportagem », en portugais ;

- chaîne a été lu comme change ( "mudança ») et de là, le lecteur est arrivé à un équivalent du système de chaîne d'argent au Brésil (« pirâmide »), car, dit-il, l'argent change de mains ;

- chômage a été traité à partir de chômeurs qui avait été traité, à son tour, à partir d'employeurs ;

- le terme offres a eu comme lecture, par un même individu, les termes «ladrões » (voleurs), « donos " (maîtres) et, finalement, «ofertas » (offres) ; de même, le terme escroquerie a été lu par le même lecteur comme « algo sujo » (quelque chose de sale), « absurdo » (absurde), « engodo » ( tromperie) et, finalement, «farsa » (farce);

dans un processus d'hypéronymisation, le terme dressés a été lu en tant que faits, ce qui résoud, d'une certaine façon, le problème pour le lecteur ;

Un autre type de traitement qui nous a surpris c'est la capacité montrée par le lecteur de gérer le processus de construction du sens en se servant des stratégies métacognitives de contrôle et d'adéquation. Ce mécanisme explique, par exemple, les différentes lectures d'un même terme jusqu'à la construction définive de son sens (exemples ci-dessus). A l'opposé, certaines hésitations et surtout certains silences ont marqué la recherche. Ainsi, des ressemblances graphiques ou sonores évidentes n'ont pas été perçues (fausses «falsas » en portugais) et certains mots ont simplement été ignorés par les lecteurs ( parquet, bidon), signe soit d'une surcharge cognitive importante soit d'une décision délibérée de ne pas s'attarder sur des mots extrêmement difficiles pour eux, ce qui viendrait encore montrer le rôle des activités métacognitives de contrôle.

Cependant, ce que cette recherche nous a surtout montré c'est l'importance - assez oubliée ces derniers temps par les études sur la lecture en langue étrangère - de l'élément formel dans le traitement lexical. Ainsi, examinons de plus près la lecture du terme " rapport » dans Un rapport gouvernemental dénonce l'augmentation des fausses offres d'emploi. Trois lecteurs ont lu ce terme comme "anúncio" (annonce en français), traduction assez inattendue pour un terme qui peut être rapproché d'autres plus fréquents en portugais ("reportagem», "relatório», par exemple). En connaissant, pourtant, les recherches sur le lexique mental, on peut donner une explication de ce traitement: les voisins orthographiques d'un terme plus transparent dans le contexte immédiat (dénonce) ont été activés avant que les lecteurs n'activent les voisins orthographiques du terme rapport. Ainsi, à partir de dénonce, ils ont activé « denúncia » et sont arrivés, ensuite, à " anúncio » (en portugais) terme choisi pour traduire rapport et qui s'adapte bien à la situation, car "anúncio » peut faire référence au verbe "anunciar ", équivalent de communiquer, de faire savoir. Un troisième lecteur est allé encore plus loin 
dans son processus de construction de sens et a proposé "comunicado» (communiqué), indiquant ainsi une complète prise en compte du contexte.

Une procédure semblable a été utilisée dans la lecture/traduction du terme escroquerie. Certains ont associé ce terme au mot anglais escroquer, utilisé en portugais, d'autres ont parcouru un long chemin, examinant chacune de ses utilisations pour arriver à la construction d'un sens convenable; d'autres encore ont associé ce terme à des item plus disponibles dans leur lexique mental, tels que saloperie et plaisanterie. Un lecteur, pourtant, a montré un comportement assez bizarre, attribuant au terme en question le sens de " farsas" (une farce). Or, escroquerie et "farsas" ce sont deux termes qui peuvent être donnés comme proches, mais dont l'approximation du point de vue formel est quand même inattendue. Cependant, quand on sait que le lecteur en question n'a pas réussi à traduire le terme fausses, on peut faire l'hypothèse que cette impossibilité est restée réservée et apparaît plus tard dans "farsas ». Il ne faut pas oublier aussi que la phrase en question (Un rapport dénonce...) gère une expectative négative qui est traduite par la lecture de fausses en tant que "farsas», car qui dénonce, dénonce un fait pour le moins irrégulier.

Chaîne dans « un système de chaîne d'argent » a provoqué, lui-aussi, des comportements très productifs qui n'ont eu lieu qu'après la rencontre, par le lecteur, d'un premier sens sur lequel il a commencé à travailler. Ce premier sens a été, pour la plupart des lecteurs, le terme "cadeia» (similaire du point de vue formel, mais un faux ami). D'autres ont construit le sens correct à partir de l'acception du terme en tant que chaîne de télévision. Ainsi, la difficile allusion à un système irrégulier de captation d'argent, les chaînes d'argent, a pu être compris grâce à l'activation par les lecteurs d'un schéma mental adéquat et la rencontre d'une forme pour les mots inconnus.

Ces exemples nous montrent, bien sûr, l'importance de l'interaction entre les stratégies descendantes et ascendantes de lecture, mais ils vont plus loin, car ils nous montrent que cette interaction n'est réussie que lorsqu'il y a prise en compte des éléments formels pendant le traitement lexical, importance qui a été niée par maints courants de lecture, surtout en langue étrangère.

La diversité des comportements et l'importance du formel nous ont emmenée à formuler une petite schématisation des comportements qui serait la suivante :

1. Quand le terme de la langue étrangère est connu du lecteur, c'est-à-dire, quand il possède déjà une localisation dans son lexique mental (connaissance d'une de ses acceptions, stabilisée par la fréquence d'emploi ou de contact), le terme est traité directement à un niveau que j'appelerai exclusivement sémantique ${ }^{11}$, par le moyen de l'analyse des contextes immédiat et global et des connaissances du monde déjà activées pendant la lecture ;

2. Quand le terme de la langue étrangère est inconnu ou peu familier du lecteur, il peut se produire un traitement que j'appelle "formel-sémantique»: en s'appuyant sur les caractéristiques formelles qu'il arrive à repérer dans le vocable en question (par sa graphie ou sa forme sonore), le lecteur essaie de lui attribuer une forme, en l'associant à un autre terme de sa langue maternelle ou à un terme d'une langue étrangère plus fréquente et donc plus disponible pour lui. J'appelle ce mécanisme formel-sémantique car, comme il a été montré par la recherche, ce processus d'attribution d'une forme est dirigé, de manière assez contraignante et pertinente, par la prise en compte du contexte mais seulement à partir de la rencontre d'une forme pour le mot traité ;

33 Ce traitement est, en fait, beaucoup plus inconscient qu'on ne pourrait l'imaginer et cela pour deux raisons : 1) par le fait déjà démontré que la lecture d'un terme lexical active 
tous ses voisins orthographiques;2) on sait aujourd'hui, grâce aux études sur les mouvements oculaires, que pratiquement tous les termes d'une phrase sont focalisés pendant la lecture. Or, pendant la lecture en langue étrangère, on a affaire à des lecteurs qui, par leur expérience en langue maternelle, effectuent involontairement un type différent de lecture, en cherchant les données de manière beaucoup plus rapide. Ainsi, le lecteur expert finit par fixer plusieurs parties du texte à la fois et il active plusieurs voisins orthographiques presque simultanément. Le résultat c'est que, parfois, mené par ce qui lui semble être le plus familier et par une prise en compte trop rapide du contexte, le lecteur étend, de façon inconsciente, la recherche de sens aux voisins orthographiques d'autres termes de la phrase qui n'étaient pas traités. Ce «déplacement» du processus constructif peut expliquer la lecture de rapport comme annonce : n'ayant pas trouvé, dans le temps nécessaire, une forme de sa langue maternelle pour rapport, la proximité du verbe dénonce active le mot "denúncia » et grâce à l'extrême intervention du contexte, le lecteur arrive à « anúncio » pris dans le sens de « anunciar » (annoncer) quelque chose.

L'approximation avec une forme quelconque de plus grande fréquence ou d'accès plus facile permet l'entrée dans le lexique mental, inaugurant ainsi un travail détaillé de stabilisation, c'est-à-dire, d'adéquation du premier sens construit au contexte (ce qui peut ne pas aboutir à un sens correct, ex. : presse-« pressa»). Bien que cette explication puisse être inversée (on peut postuler que c'est la charge sémantique résultant de la lecture, impliquant aussi la prise en compte des éléments extra-textuels, qui fait que le lecteur construise le sens du vocable inconnu) nous croyons que l'existence d'un appui formel est nécessaire pour que cela arrive. On ne peut pas reconnaitre un mot sans l'identifier avant; il faut que l'on parte d'un point quelconque. Comme l'affirme Singleton (1994), quand on voit un mot de la langue maternelle que l'on ignore, des associations parfois invraisemblables sont établies, par approximation sonore ou graphique. Ainsi, le lecteur débutant - dans une stratégie de cohérence par rapport à la tâche de lecture qu'il doit effectuer - associe au maximum le terme inconnu aux éléments de son lexique mental.

Nous pouvons dire que, dans le cas du français/portugais, cet appui formel ne peut pas ne pas exister, surtout dans les premiers moments de l'apprentissage. Au fur et à mesure qu'il progresse, l'étudiant montre la constitution d'un lexique mental en français (observé grâce aux associations dans la langue étrangère) qui doit devenir de plus en plus autonome, automatisé et constitué d'éléments sémantiques, conceptuels et culturels approfondis.

Pendant le traitement formel-sémantique, nous distinguerions donc deux moments :

1. L'attribution d'une forme au mot inconnu comme résultat d'une décodification graphophonologique et du travail sémantique contextuel, et la postérieure activation d'un premier sens ; le lecteur amorcerait ainsi le processus de construction du sens ;

2. L'activation d'un premier sens ne veut pas dire la construction du sens définitif ou le plus adapté au contexte (car la lecture est toujours un mécanisme flexible et passible d'adaptations constantes). Elle aurait lieu dans un deuxième temps, où la pertinence de la sélection d'un candidat serait alors analysée par rapport au contexte de façon plus précise. Ce serait la phase finale de construction du sens.

Identifier formellement un mot serait, ainsi, trouver un chemin - n'importe quel chemin - pour donner l'entrée dans le lexique mental. Reconnaître serait alors donner suite à ce processus en construisant un sens, non en fonction, mais par la considération des données prises dans les contextes immédiat et global. Dans plusieurs cas, il peut y avoir identification mais non reconnaissance. Ce processus n'est pourtant pas toujours 
fructueux, car il peut y avoir le maintien de la forme et une postérieure reconnaissance sémantique dans d'autres occurrences du terme (c'est l'exemple donné de fausses). Il est important de souligner dans ce type de traitement l'importance de la prosodie et des traits phonologiques pendant le processus d'identification, ce qui signifie que la transparence sonore est aussi importante que la transparence graphique, surtout en langue étrangère.

Ces conclusions mettent en question le rôle du contexte dans la reconnaissance des mots. Certains auteurs, tels Alegria (1991) e Perfetti $(1982,1989)$ dans leurs études sur l'acquisition de la langue maternelle, le rejettent totalement. Cette position doit être nuancée en langue étrangère, car on a affaire à des lecteurs experts mais qui ne disposent pas d'un bagage linguistique suffisant et autonome de la langue étrangère qu'ils apprennent (à l'inverse de ce qui se passe en langue maternelle où le bagage oral est déjà important) Ainsi, il nous semble que la position de Pynte (1983) qui accepte le traitement du contexte dans le processus de reconnaissance des mots est plus raisonnable. A partir des études faites avec des mots polysémiques isolés et en contexte, cet auteur conclut que le contexte est déterminant surtout dans la deuxième phase de la lecture, c'est-à-dire, pendant l'interprétation et la construction finale du sens. Dans cette étude, l'auteur insiste sur le fait que l'accès lexical ne peut pas être vu de manière indépendante ni des mécanismes de perception, ni du traitement du contexte dans lequel le mot est inséré.

Dans cette perspective, la signification d'un mot ne serait pas totalement comprise dans le lexique. Elle serait attribuée grâce au travail avec d'autres termes de l'énoncé et du texte lui-même, ce qui nous fait souligner l'expérience de Carr (1981), déjà citée ici, selon laquelle les sujets plus experts maintiennent différentes interprétations d'un item ambigu jusqu'à sa complète interprétation, pendant que les sujets plus faibles semblent procéder à un choix bien plus tôt, prenant ainsi le risque de se tromper.

40 En d'autres mots, on peut dire que les bons lecteurs, ayant accédé correctement au lexique mental, peuvent alors construire de façon plus productive le sens le plus convenable au mot en question, en se servant des contraintes et des données du contexte. Dans la théorie énonciative, les auteurs prennent garde de ne jamais utiliser le contexte comme un élément capable de défaire les ambiguïtés de sens. En fait, chaque terme employé contient en soi des propriétés qui déclenchent tel ou tel contexte particulier. De là résulte le processus de construction du sens. Il n'y a pas d'intervention du contexte comme quelque chose qui vienne suppléer ou expliquer la signification d'un terme, car le terme lui-même s'explique dans son emploi.

41 L'analyse proposée pour le traitement formel-sémantique renforce aussi la notion de lecture comme un processus d'activation-diffusion ou de construction-intégration, tel qu'il est proposé dans le dernier modèle élaboré par Kintsch et Kintsch (1991).

42 En ce qui concerne le traitement essentiellement sémantique, il a lieu quand existe déjà la connaissance d'une des acceptions du terme lu, ou encore, dans un cas complètement inverse, quand il y a difficulté de travail sur le texte proprement dit. Ce mécanisme, bien qu'intéressant dans certaines tâches de lecture, ne mène pas l'étudiant sur la voie de l'apprentissage, sauf au prix d'une grande charge cognitive : s'il n'y a pas de perception formelle du terme inconnu, il ne peut y avoir de mémorisation et, à chaque nouvelle occurrence, le lecteur doit employer des stratégies coûteuses pour construire son sens. Hulstijm (1994), dans une étude sur l'acquisition incidente du lexique pendant la lecture en langue étrangère, confirme ce fait en montrant que les apprenants de langue 
étrangère ont plus de chance d'inférer un mauvais sens pour un mot inconnu quand ils ne le traitent pas de façon concrète.

En outre, plusieurs auteurs (Souchon, 1992, Trevise, 1994) affirment que, dans l'impossibilité d'un travail plus approfondi du texte, le lecteur finit par activer une connaissance minimale du sujet et ils observent qu'en l'absence de compréhension de nombreuses marques linguistiques, une représentation sémantique, à caractère propositionnel, est directement construite. Cette construction a lieu en fonction aussi bien des modèles mentaux du lecteur, de ses attentes, de ses anticipations et inférences, qu'en fonction d'une certaine cohérence pragmatique de la tâche de lecture.

Comme nous avons essayé de le montrer, les types de traitement lexical sont, ou doivent être, la première phase de la construction du sens qui a besoin d'une suite pour être considérée pertinente et, même, passible d'acquisition. Il est important de souligner que, pris de façon exclusive, ces traitements peuvent gérer de graves fautes de compréhension dues aussi bien à l'homophonie, à la similitude des termes de la langue maternelle et de la langue étrangère, qu'à l'interférence exagérée des connaissances du monde. Il ne faut pas oublier que, comme il est psychologiquement difficile de mettre en question ses propres croyances, l'apprenant préfère en général deviner le sens d'un terme, voire d'un texte, en fonction de ses hypothèses, au lieu de modifier ce qui est déjà établi en lui.

En langue maternelle, on l'a déjà dit, les recherches montrent que les bons lecteurs sont justement ceux qui savent contrôler constamment leurs lectures, qui parcourent le texte dans plusieurs directions, qui se posent des questions. Les lecteurs faibles, de leur côté, ne possèdent pas cette flexibilité de traitement et adoptent un comportement passif dans l'approche des textes.

Un autre point intéressant, c'est que l'on observe que le lecteur est guidé par une notion de proximité entre les langues et par un sens de l'économie et du coût cognitif. Il consacre un traitement approfondi aux items qu'il considère comme les plus importants. Ce sont des aspects fondamentaux quand il s'agit de la lecture en langue étrangère dans une situation scolaire et qui résultent, en fait, du transfert de comportements déjà existants dans la langue maternelle.

Sur ce sujet, Giacobbe (1989) affirme qu'étant donnée la condition dans laquelle se trouve un apprenant de langue étrangère (qui possède déjà la maîtrise du langage et connaît son fonctionnement, mais à qui manquent des éléments linguistique dans la langue-cible) son interlangue est à la fois dynamique et systématique. Cette dernière caractéristique vient $\mathrm{du}$ fait qu'en essayant de pallier son manque de bagage linguistique, l'apprenant d'une langue étrangère élabore constamment des hypothèses sur elle, hypothèses qui ne sont pas toujours cohérentes ou homogènes, mais qui constituent un système, ou un ensemble de micro-systèmes plus ou moins provisoires, avec une tendance importante à des généralisations de plus en plus grandes et à une stabilisation. Giacobbe conclut ses recherches en affirmant que le recours à la langue maternelle, effectué par l'apprenant, est systémique et opératoire. Il est systémique dans la mesure où l'apprenant a dans la langue maternelle une source de systématisation, d'organisation, qui lui permet d'ordonner, à son tour, l'immense quantité de données dispersées reçues pendant l'apprentissage de la langue étrangère. Il est opératoire dans la mesure où l'approximation entre les deux langues doit se traduire par des hypothèses opératoires qui, en transformant les formes extraites de la langue maternelle, permettent de les intégrer à son interlangue. Giacobbe insiste sur le fait que ce n'est pas l'apprentissage qui "guérit » l'étudiant de l'influence de sa langue maternelle, mais au contraire, c'est le 
recours à cette langue qui constitue une des conditions de l'apprentissage. Éviter ce recours serait aller contre un processus naturel de l'élève, la construction de sa propre interlangue, qui est l'une des bases du nouveau système en cours de construction par l'apprenant: l'approximation constante entre les langues source et cible crée un système d'hypothèses opératoires responsables de la bonne évolution de l'apprentissage. C'est pour cela que l'analogie formelle même erronée, ne doit pas être vue comme négative, mais plutôt comme un passage vers l'autonomie du traitement lexical. L'important c'est que cette utilisation de la langue maternelle et l'appui formel qui en résulte soient constamment contrôlés par une attitude de réflexion et de distance de l'apprenant.

\section{Des écluses de sens}

Pour conclure cet article, nous proposons ici une image qui peut illustrer ce qui, selon nous, se produit pendant la lecture en langue étrangère: des lecteurs experts, qui ont déjà des habitudes de lecture et qui «cherchent » donc sans cesse (et de façon parfois inconsciente) le sens dans un texte, agissent devant lui comme s'ils étaient devant le tableau de contrôle d'un complexe système d'écluses. Guidés par leurs connaissances langagières, ces lecteurs dirigent (activent) l'ouverture d'une ou de plusieurs portes à la fois ; avec l'entrée de l'eau, du (des) sens, immédiatement de nouveaux mécanismes sont activés, de nouvelles portes sont ouvertes et inondées, pendant que d'autres se vident, laissant cependant les marques de ce processus dans l'activation de nouveaux éléments. Dans un flux constant, simultané et généralement inégal et hétérogène, tout le système est actionné jusqu'à se stabiliser à la limite établie par l'opérateur du système (notre lecteur...), qui, tout en le contrôlant, est directement impliqué dans son fonctionnement.

De la même manière, le sens est construit dans le texte par le moyen d'activations, de diffusions et de désactivations des éléments pas toujours homogènes, de même relief et importance, consécutifs ou hiérarchisés, mais toujours perçus et traités en fonction des « ancrages » qui y sont faits par le lecteur. Ces ancrages sont le résultat de l'habileté de lecture du sujet, de son apprentissage, de ses connaissances. Dans ce mécanisme complexe, la matérialité et la virtualité des éléments actionnés sont prises en considération.

Malgré la constante impression d'indicible et d'inaccessible, quelques processus mis en oeuvre lors de la lecture ont pu être démontrés. Le principal a été, pour nous, le traitement lexical pour lequel on peut établir, de façon résumée, trois phrases principales :

1. identification d'une forme comme «mot de passe » pour l'amorçage du traitement ;

2. accès à une acception première, de connaissance et fréquence plus grandes pour le lecteur ;

3. construction d'un sens définitif à partir du travail d'inférence auprès des contextes immédiat et global, et de l'exercice de contrôle et d'activité métacognitive qui stabilisent la construction faite.

51 Ces trois phases s'emboîtent et sont complémentaires : plus grande est la sensibilité développée en (3), plus importante sera l'attention portée à la forme du mot (1) et à son signifié (2). Dans ce processus, de nouveaux termes sont appris et mémorisés ce qui, à son tour, amène l'étudiant à comparer davantage sa langue maternelle avec la langue étrangère-cible, perfectionnant ainsi ses capacités de contrôle et renouvelant le cycle. 

construction du sens et son bagage sémantique qu'il essaie d'« adapter » à la lecture d'une langue dont il maitrise mal les éléments. Dans ce processus, il doit être amené à disposer d'un nombre chaque fois plus grand de stratégies pour augmenter ainsi son éventail de possibilités de succès. C'est un des rôles principaux que j'attribue à l'enseignement d'une langue étrangère. L'étudiant doit être mis en contact avec une grande quantité d'écrit, doit être amené à affronter ces écrits sans peur et doit être aussi confronté à des lectures de conflit qui, en respectant un certain seuil de difficulté, l'aideront à adopter des comportements de plus en plus productifs et pertinents. Plus il y aura de l'inattendu, de la surprise, du conflit, plus les chemins de sens seront hétérogènes et porteurs d'apprentissage. C'est une demande aussi pour que l'étudiant de langue étrangère ne soit privé de rien: l'oral, l'écrit, la langue maternelle, d'autres langues étrangères, des techniques d'approche globale, mais aussi des techniques « simples» de connaissance et apprentissage du lexique, tout cela est important dans l'établissement d'un réseau de plus en plus étendu de sens et de possibilités de traitement. Plus il y aura de chemins à parcourir, plus nombreuses seront les réussites en lecture. Et plus la lecture valorisera l'indicible...

\section{BIBLIOGRAPHIE}

ALEGRIA, J. (1991) Mécanismes spécifiques de la lecture, in Les entretiens Nathan : Lecture (Actes I), Paris, Nathan.

CICUREL, F., MOIRAND, S. (1990) Apprendre à comprendre l'écrit : hypothèses didactiques, in GAONAC'H, D. (Org.) - Acquisition et utilisation d'une langue étrangère, l'approche cognitive, Paris, Hachette, pp. 147-158.

FAYOL, M. (1992a) Comprendre ce qu'on lit : de l'automatisme au contrôle, in FAYOL, GOMBERT et alii (1992) - Psychologie Cognitive de la lecture, Paris, PUF, pp. 73-106. 
FAYOL, M. (1992b) La compréhension lors de la lecture : un bilan provisoire et quelques questions, in LECOCQ, P. (ed.), (1992) - La lecture : processus, apprentissage, troubles, Lille, PUL, pp. 79-102.

GAONAC'H, D. (org.), (1990) Acquisition et utilisation d'une langue étrangère, l'approche cognitive, Paris, Hachette.

GIACOBBE, J. (1989) Construction des mots et construction du sens : cognition et interaction dans l'acquisition du français par des adultes hispanophones, Thèse de Doctorat en Linguistiques, Université Paris VII.

HULSTIJN, J. H. (1994) L'acquisition incidente du lexique en langue étrangère au cours de la lecture : ses avantages et ses limites, Aile, no 3, pp. 77-96.

KINTSCH, W., KINTSCH, E. (1991) La compréhension de textes et l'apprentissage à partir de textes : la théorie peut-elle aider l'enseignement?, in Les entretiens Nathan : lecture (actes I), Paris, Nathan, pp. 13-28.

LECOCQ, P. (ed.), (1992) La lecture : processus, apprentissage, troubles, Lille, PUL.

LE NY, J.-F. (1989) Science cognitive et compréhension du langage, Paris, PUF.

MANGUEL, A. (1997) Uma história da leitura, São Paulo, Companhia das Letras, p. 36. Edition originale: A history of reading, Londres, Harper Collins, 1996.

MINSKY, M. (1975) A framework for representing knowledge, in WINSTON (ed.) - The psychology of computer vision, NY, MacGraw Hill.

PERFETTI, C. (1982) Contexte discursif, identification de mots et capacité de lecture, Bulletin de Psychologie, Tome XXXV, no 356, pp. 571-578.

PERFETTI, C. (1989) Représentations et prise de conscience au cours de l'apprentissage de la lecture, in RIEBEN, L, PERFETTI, C. (dir.) - L'apprenti lecteur, Neuchâtel, Paris, Delachaux \& Niestlé, pp. 61-84.

PIETRARóIA, C. (1996) Léxico, leitura e construção do sentido em Francês Língua Estrangeira, Thèse de Doctorat, São Paulo, FFLCH-USP.

PYNTE, J. (1983) Lire...identifier comprendre, Lille, PUL,.

SANFORD, A.J., GARROD, S. (1982) Vers la construction d'un modèle psychologique de la compréhension du langage écrit, Bulletin de Psychologie, no 356, pp. 643-648.

SAVEE, E. « La mycorhize », in Nouvelles Nouvelles, publication trimestrielle de la Fondation David Kupfermann, Paris, 1990

SAUSSURE, F. (1916) Cours de Linguistique Générale, Paris, Payot, $3^{\mathrm{a}}$ ed. : 1972.

SCHANK, R.C., ABELSON, R. P. (1977) Scripts, pans, goals and understanding: an inquiry into human knowledge structures. Hillsdale, Lawrence Erlbaum.

SEGUI, J. (1992) Les composantes cognitives de la lecture, in Lecocq (Ed.) - La lecture : processus, apprentissage, troubles, Lille, PUL.

SINGLETON, D. (1994) Introduction : le rôle de la forme et du sens dans le lexique mental en L2, Aile, no 3, pp. 3-27.

souchon, M. (1992) Compétences, savoirs, représentations. Leur rôle dans la lecture en L2 en début d'apprentissage. Le cas de deux langues-cultures « voisines », Cahiers du Crelef, n 32, pp. 107-136. 
TREVISE, A. (1992) La gestion cognitive de l'étrangeté dans l'acquisition d'une langue étrangère, Aile, $\mathrm{n}^{\circ}$ 1, pp. 87-106.

ZAGAR, D. (1992) L'évaluation de la lecture : comment construire un instrument d'évaluation diagnostique ?, in LECOCQ, P. (ed.) - La lecture : processus, apprentissage, troubles, Lille, PUL, pp. 213-230.

ZAGAR, D., JOURDAIN. C. et LÉTÉ, B. (1993) Eccla : Evaluation-diagnostic des Capacités Cognitives du Lecteur Adulte. Paris, Centre National de Documentation Pédagogique.

\section{ANNEXES}

LIBÉRATION mardi 9 novembre 1994

\section{EMPLOI}

\section{Un rapport gouvernemental dénonce l'augmentation des fausses offres d'emploi}

L'EXPLOSION du chômage a entraîné une floraison d'offres d'emploi « bidon ». A l'instar des associations de chômeurs et de consommateurs les services du ministère de l'Economie chargés de la consommation ont constaté depuis plusieurs mois une « recrudescence des plaintes » contre les « fausses offres d'emploi ». Depuis la fin 1993, 80 procès verbaux ont été dressés et trois rapports pour escroquerie transmis au parquet, révèle un bilan d'étape publié hier par le ministre de l'Economie Edmond Alphandéry.

Trop souvent, les chômeurs se voient ainsi demander de verser une participation financière sans « la moindre garantie », sur le sérieux des offres. C'est le cas des offres « d'activité indépendante à domicile », où l'on demande de verser une somme qui peut aller jusqu'à 500 francs pour recevoir un « manuel » détaillant les travaux à effectuer (fabrication de bijoux fantaisie, de jouets en peluche, etc.).

«En réalité, soit ils ne reçoivent pas la brochure, soit ils ne sont jamais payés pour le travail effectué », explique le ministère.

Autre escroquerie, celle des offres d'emploi apparemment classiques qui servent en fait de paravent à des « systèmes de chaînes d'argent ». Le principe est connu : après avoir acquitté un «droit d'entrée au réseau » la personne doit prospecter par petites annonces, touchant une commission à chaque nouvelle inscription.

Des « marchants de listes » vendent aux chômeurs une liste d'employeurs potentiels. Mais " en réalité, les sociétés susceptibles d'embaucher n'ont jamais été consultées par cet intermédiaire . » S'agissant d'offres d'emploi Minitel, l'escroquerie consiste à proposer les mêmes offres sur des serveurs différents, à ne pas réactualiser des fichiers ou à « repiquer » des offres parues dans la presse. (AFP).

\section{NOTES}

1. SAVEE, E. - «La mycorhize », in Nouvelles Nouvelles, publication trimestrielle de la Fondation David Kupfermann, Paris, 1990, no 18, p. 39.

2. MANGUEL, A. (1997) - Uma história da leitura, São Paulo, Companhia das Letras, p. 36. Edition originale : A history of reading, Londres, Harper Collins, 1996. 
3. Rien n'est simple quand on parle du langage...

4. Zagar, D., Jourdain. C. et Lété, B. (1993) - Eccla : Evaluation-diagnostic des Capacités Cognitives du Lecteur Adulte. Paris, Centre National de Documentation Pédagogique.

5. Pour une révision, voir ZAGAR, D. (1992) - L'évaluation de la lecture : comment construire un instrument d'évaluation diagnostique?, in LECOCQ, P. (ed.), La lecture: processus, apprentissage, troubles. Presses Universitaires de Lille.

6. « Dans le domaine particulier du français langue étrangère, tel qu'il s'est développé en France, depuis le début des années 1960, c'est au milieu des années 1975 que l'on a commencé à s'intéresser à la lecture du sens. Mais s'il y a eu quelques idées venues de la connaissances des modèles psycholinguistiques afin de justifier certaines pratiques, les méthodologies proposées aux apprenants pour comprendre l'écrit sont dues, semble-t-il, à de bonnes intuitions, productives certes mais de nature empirique. Il n'y a pas eu, à notre connaissance, de recherche effective s'appuyant sur des modèles théoriques construits à cet effet, pas plus que de recherche d'envergure de nature expérimentale, en français langue étrangère. » (Coste 1974) (p. 147).

7. Cf. PIETRARÓIA, C. (1996) - Léxico, leitura e construção do sentido em Francês Língua Estrangeira, Thèse de Doctorat, São Paulo, FFLCH-USP.

8. «Emploi : un rapport gouvernemental dénonce les fausses offres d'emploi. ». Ce texte a été transcrit dans l'annexe de cet article.

9. FAYOL, M. (1992b :93). Les termes soulignés sont en italiques dans le texte original.

10. Ces deux types de mises en relation (formelle et sémantique) avaient déjà été suggérées par Saussure dans le chapitre "Rapports syntagmatiques et rapports associatifs» du Cours de Linguistique Générale : En dehors du discours, les mots offrant quelque chose de commun s'associent dans la mémoire, et il se forme ainsi des groupes au sein desquels règnent des rapports très divers ". L'auteur donne comme exemple le mot enseignement qui peut susciter une «multitude» (terme de l'auteur) d'autres mots : enseigner, renseigner, ou bien armement, changement, ou encore éducation, apprentissage. La multitude des mots qui s'associent s'explique par le fait que ce type de coordination se trouve dans le cerveau sous forme virtuelle (elles font partie de ce trésor intérieur qui constitue la langue chez chaque individu). Les rapports peuvent être morphologiques, sémantiques ou sonores. La conclusion de Saussure à ce sujet, mérite d'être transcrite : Donc il y a tantôt communauté double du sens et de la forme, tantôt communauté de forme ou de sens seulement. Un mot quelconque peut toujours évoquer tout ce qui est susceptible de lui être associé d'une manière ou d'une autre.

11. Il faut bien préciser que la distinction faite entre formel et sémantique n'est valable que pour une bonne compréhension des traitements lexicaux effectués. Elle ne nie pas la constitution de tout mot comme forme et sens.

\section{RÉSUMÉS}

La difficulté de toute étude portant sur la compréhension écrite vient de la grande quantité de paramètres et de variables en jeu. Vue comme activité de communication, construction et représentation du sens, la lecture se caractérise par des mécanismes difficilement explicitables, car on a affaire à des représentations langagières spécifiques à chaque lecteur dans des situations et des contextes eux aussi bien déterminés. Pourtant, cela ne nous a pas empêchée d'élaborer une recherche sur la lecture en langue étrangère ayant comme objectif de «saisir » les mécanismes 
de construction du sens. Ce sont les résultats de cette recherche, portant principalement sur le traitement lexical en langue étrangère, qui sont présentés dans cet article.

This paper analyses the specificities of the process of construction of the meaning through the activity of reading texts in a foreign language.

\section{AUTEUR}

\section{CRISTINA CASADEI PIETRARÓIA}

Université de São Paulo (USP) 